\title{
Obstruktive Atemwegserkrankungen als Berufskrankheiten
}

\author{
Obstructive Airway Disorders Representing Occupational Diseases
}

Autor

Institut

\section{Baur}

Ordinariat und Zentralinstitut für Arbeitsmedizin und Maritime Medizin Hamburg (Institutsleiter: X. Baur) eingereicht 14.8. 2011 akzeptiert nach Revision 13. 9.2011

Bibliografie

DOI http://dx.doi.org/ 10.1055/s-0031-1291396 Pneumologie 2011; 65: 654-661 (c) Georg Thieme Verlag KG Stuttgart · New York ISSN 0934-8387

Korrespondenzadresse: Prof. Dr. med. Xaver Baur Ordinariat und Zentralinstitut für Arbeitsmedizin, Universitätsklinikum HamburgEppendorf Seewartenstraße 10 20459 Hamburg baur@uke.de

\section{Zusammenfassung \\ $\nabla$}

Obstruktive Atemwegserkrankungen, das Asthma bronchiale und die COPD, haben eine Prävalenz von jeweils etwa $5 \%$ in unserer Bevölkerung. 5 $25 \%$ der Fälle gehen auf die Einwirkung von Schadstoffen in der Arbeitswelt zurück. Dabei sind pathophysiologisch und nach dem Berufskrankheitenrecht allergische Krankheitsbilder (BK-Nr.4301) von solchen chemisch irritativer Genese (BK-Nr.4302; überwiegend auch BK-Nr. 1315) und durch anorganische Stäube verursachten (BK-Nr.4111) zu unterscheiden. Unter den ursächlichen Noxen dominiert derzeit noch Steinkohlengrubenstaub, gefolgt von Mehl- und Backmitteln, Schweiß-, Gieß- und Schneidrauchen sowie Isocyanaten. Die Prognose ist in der Mehrzahl der Fälle schlecht und abhängig von der Dauer der Schadstoffeinwirkung. Dies weist auf die Dringlichkeit der Frühdiagnose und der gezielten Individualprävention hin. Die neue internationale GHS-Kennzeichnung gesundheitsschädlicher Arbeitsstoffe (EU: CLP) betrifft auch inhalative Gefahrstoffe. Sie löst die bisherigen R- und S-Sätze durch die Gefahrenhinweise H (H-Sätze, Hazard Statements) und P-Sätze (Precautionary Statements) sowie neue Gefahrenpiktogramme ab.

\section{Einleitung}

$\nabla$

Etwa jede sechste obstruktive Atemwegserkrankung geht auf arbeitsbedingte Noxen zurück [1 3]. Hierzu zählen anorganische Stäube, wie sie im Bergbau auftreten [4-7], über 250 Allergene und nahezu ebenso viel atemwegsirritative Stoffe (Übersichten: [8-10]).

Pathogenetisch ist von Bedeutung, dass Aerosole mit einem Durchmesser von über $10 \mu \mathrm{m}$ überwiegend im Bereich der Nase infolge der dortigen Atemstromturbulenzen deponiert werden. Aerosole mit kleinerem Durchmesser erreichen in zu-

\section{Abstract \\ $\nabla$}

Obstructive airway diseases, i.e., bronchial asthma and COPD, have a prevalence of about $5 \%$ each in our population. 5 to $25 \%$ of the cases are caused by the impact of hazardous substances in workplace atmospheres. Pathophysiologically and according to legal definitions on occupational diseases, allergic disorders (occupational disease no 4301) have to be differentiated from those of chemically irritative genesis (occupational disease no 4302 and predominantly also no 1315) and from afflictions in coal miners induced by inorganic dust (occupational disease no 4111). At present, hard coal mine dust is still the prevailing noxious agent followed by flour/baking additives, fumes occurring during welding, casting and cutting procedures, and isocyanates. In the majority of cases, the prognosis is not good and depends on the duration of exposure to the causative impact. This emphasises the urgency of early diagnosis and targetted individual prevention. The new international labelling of working materials hazardous to health (GHS; EU: CLP) also refers to inhalative noxae. It replaces the hitherto valid $\mathrm{R}$ und $\mathrm{S}$ notes by hazard statements $(\mathrm{H})$ and precautionary statements $(\mathrm{P})$ as well as by new hazard pictograms.

nehmendem Maße die tieferen Atemwege und die Alveolen und werden dort abgelagert. Gase mit guter Wasserlöslichkeit werden großteils bereits im Bereich der oberen Atemwege absorbiert, wenig oder nicht wasserlösliche erreichen dagegen ganz überwiegend die Alveolen.

Inhalativ aufgenommene anorganische, im Bereich der Atemwege und der Alveolen deponierte Stoffe induzieren über zugrunde gehende Makrophagen, Zytokinfreisetzungen und Bildung reaktiver Sauerstoffradikale persistierende inflammatorische und toxische Effekte mit langsam progredienten irreversiblen Veränderungen im Atem- 
Tab. 1 Legaldefinitionen der obstruktiven Berufskrankheiten.

\begin{tabular}{|ll}
\hline BK-Nr. 4301 & $\begin{array}{l}\text { Durch allergisierende Stoffe verursachte obstruktive } \\
\text { Atemwegserkrankungen (einschließlich Rhinopathie), } \\
\text { die zur Unterlassung aller Tätigkeiten gezwungen haben, } \\
\text { die für die Entstehung, die Verschlimmerung oder das } \\
\text { Wiederauftreten der Krankheit ursächlich waren oder } \\
\text { sein können. }\end{array}$ \\
\hline BK-Nr. 4302 & $\begin{array}{l}\text { Durch chemisch-irritativ oder toxisch wirkende Stoffe } \\
\text { verursachte obstruktive Atemwegserkrankungen, die zur } \\
\text { Unterlassung aller Tätigkeiten gezwungen haben, die für } \\
\text { die Entstehung, die Verschlimmerung oder das Wieder- } \\
\text { aufleben der Krankheit ursächlich waren oder sein kön- } \\
\text { nen. } \\
\text { Erkrankungen durch Isocyanate, die zur Unterlassung } \\
\text { aller Tätigkeiten gezwungen haben, die für die Entste- } \\
\text { hung, die Verschlimmerung oder das Wiederauftreten } \\
\text { der Krankheit ursächlich waren oder sein können. }\end{array}$ \\
\hline Chronische obstruktive Bronchitis oder Emphysem von \\
Bergleuten unter Tage im Steinkohlebergbau bei Nach- \\
weis einer kumulativen Dosis von in der Regel 100 Fein- \\
staubjahren [(mg/m³)x Jahre]
\end{tabular}

trakt, die häufig mit einer kombinierten Ventilationsstörung einhergehen [11,12]. Dagegen verursachen Expositionen gegenüber irritativen und toxischen Noxen typischerweise akut Symptome und eine Bronchialobstruktion. Allergische Reaktionen der Atemwege werden fast ausnahmslos durch groß-molekulare inhalativ aufgenommene Substanzen, zumeist Proteine, hervorgerufen. Sie weisen eine mindestens mehrwöchige Sensibilisierungsphase auf, während der sich spezifische IgE-Antikörper bilden.

Neue Untersuchungen weisen darauf hin, dass primäre Barrierestörungen der Haut- und Schleimhäute, so der Tight junctions und des regulatorisch wirkenden Filaggrins, Fremdsubstanzen aus der Umwelt vermehrt in die Haut und Schleimhaut eindringen lassen, wodurch Wachstumsfaktoren generiert sowie immunkompetente und inflammatorische Zellen aktiviert werden $[11,14]$. Daraus resultiert ein alle Schichten der Atemwege involvierender Remodeling-Prozess mit chronischer Inflammation, einem Zyklus aus Gewebeschädigung und Reparaturmechanismen, schließlich komplexen Veränderungen der Atemwegsstruktur. Hervorzuheben sind morphologisch erkennbare Schädigungen der Epithelzellen bis hin zu ihrem völligen Verlust, Schleimdrüsenhyperplasie, subepitheliale Verdickung, Verbreiterung der glatten Atemwegsmuskulatur, eine Metaplasie der Becherzellen, verstärkte Angiogenese und Veränderungen der extrazellulären Matrixkomponenten. Diese morphologischen Auffälligkeiten sind sowohl in den großen als auch kleinen Atemwegen und dem umgebenden peribronchialen Gewebe festzustellen [15]. Ihre klinisch-funktionellen Korrelate sind die persistierende bronchiale Hyperreaktivität sowie eine passagere, teilweise auch fixierte Bronchialobstruktion.

\section{Berufskrankheiten-Definitionen \\ $\nabla$}

In $\odot$ Tab. 1 ist der Wortlaut der vier wesentlichen, die Atemwege betreffenden Berufskrankheiten wiedergegeben. Hierbei wird nicht der Begriff Asthma verwendet, sondern der übergeordnete Terminus „obstruktive Atemwegserkrankungen“. Dieser umfasst auch die arbeitsbedingte COPD, wodurch die Zuordnung von Zwischenformen und Übergängen unproblematisch ist. Eine weitere Besonderheit ist, dass die BK-Nr.4301 die allergische Rhinitis einschließt. Die BK-Nr.1315 geht ebenfalls über obstruktive Atemwegserkrankungen hinaus und inkludiert neben der Rhinitis auch die seltene exogen allergische Alveolitis durch Isocyanate. Hervorzuheben ist ferner bei den BK-Nrn. 4301, 4302 und 1315 der einschränkende konkretisierende Nebensatz: „....Erkrankungen ..., die zur Unterlassung aller Tätigkeiten gezwungen haben, die für die Entstehung, die Verschlimmerung oder das Wiederauftreten der Krankheit ursächlich waren oder sein können“. Dieser fordert für die Anerkennung und Entschädigung durch den Unfallversicherungsträger die Aufgabe der ursächlichen Tätigkeit (Exposition). Dadurch soll die Einstellung der Krankheitsursache (Tätigkeit, Exposition) initiiert und die Chronifizierung und Verschlimmerung des Leidens verhindert werden (siehe hierzu weitere Ausführungen unter „Prognose“).

Die nach hoher langjähriger inhalativer Belastung durch anorganische Stäube hervorgerufene COPD ist im deutschen Berufskrankheitenrecht bisher auf den Steinkohlenbergbau beschränkt (BK-Nr.4111); diese Beschränkung ist nicht plausibel [16]. Neuere Studien zeigen ein erhöhtes COPD-Risiko und eine erhöhte COPD-Mortalität u.a. infolge hoher Expositionen gegenüber quarzhaltigen Stäuben, Ölnebel, Schweißrauche $[17,18]$.

\section{Prävalenz und aktuelle Statistiken \\ $\nabla$}

Bezüglich der Verursacher arbeitsbedingter obstruktiver Atemwegserkrankungen ergeben sich zwischen verschiedenen Ländern u.a. in Abhängigkeit von den industriellen Schwerpunkten und den z.T. erheblich differierenden Rechtsnormen Abweichungen [19]. So dominieren unter den Verursachern des Berufsasthmas in England Isocyanate, Mehl/Getreidestaub ( $\bullet$ Abb.1), in Südafrika Latex und Isocyanate [20] ( $\bullet$ Tab.2). In Deutschland steht derzeit noch infolge der Änderungen der Berufskrankheitenfestlegung (BK-Nr.4111 seit 1997, Aufhebung der Rückwirkungs-Klausel) mit Berücksichtigung von „Altlasten“ Steinkohlengrubenstaub ganz im Vordergrund, gefolgt von Mehl/Backwaren, Schweiß-, Gieß- und Lötrauch, Isocyanaten. $\bullet$ Abb. 2 gibt die aktuellen diesbezüglichen Berufskrankheiten-Daten für Deutschland wieder. In $\bullet$ Tab. 3 sind sowohl die angezeigten als auch bestätigten Fälle wiedergegeben.

Im internationalen Vergleich der Berufsasthmaerkrankungen liegt Deutschland im Mittelfeld mit etwa 50 Fällen/Million/Jahr [19].

\section{Neue Kennzeichnung von Gefahrstoffen}

\section{$\nabla$}

Atemwegssensibilisierende Arbeitsstoffe

In Deutschland ist die Technische Regel TRBA/TRGS 406 rechtsverbindlich, in deren Anlage die aktuellen arbeitsmedizinisch relevanten atemwegssensibilisierenden Arbeitsstoffe auflistet werden, und zwar unter Berücksichtigung der TRGS 907, der EGRichtlinie 2000/54/EG, Anhang I der Richtlinie 67/548/EWG und der Erkenntnisse der MAK- und BAT-Werte-Kommission $(\bullet$ Tab.4, $\odot$ Tab. 5). Die Senatskommission zur Prüfung gesundheitsschädlicher Arbeitsstoffe (MAK- und BAT-Werte-Kommission) aktualisiert jährlich die Liste gesundheitsgefährdender Arbeitsstoffe, wozu auch atemwegssensibilisierende gehören. - Tab. 4 fasst die aktuellen, entsprechend gekennzeichneten Substanzen zusammen, die jeweils unter Berücksichtigung der vorliegenden Literatur als „Sa“ eingestuft wurden. 


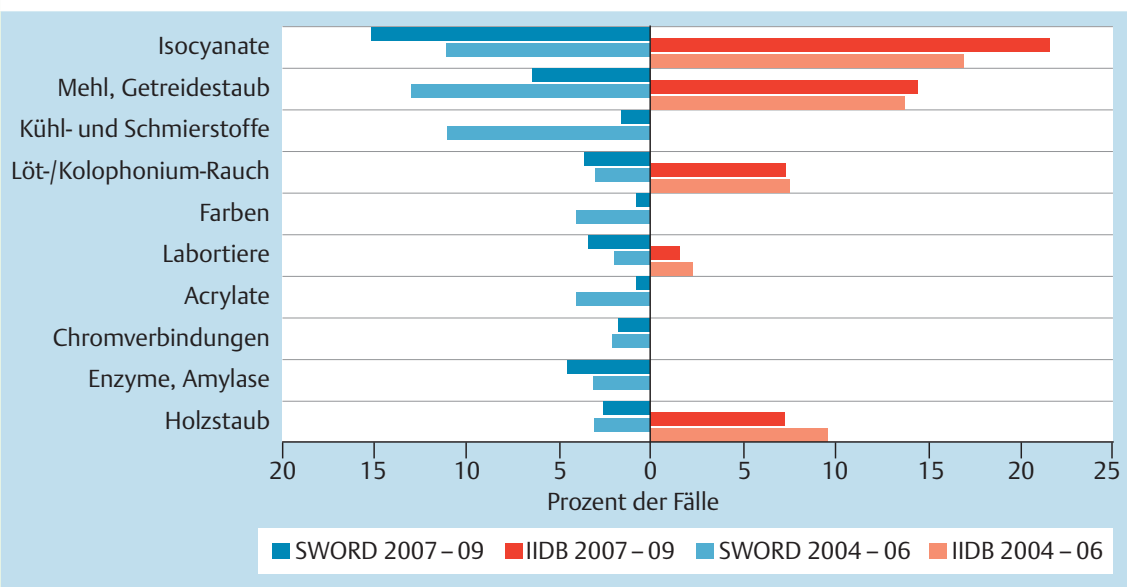

Abb. 1 Häufigste Berufsasthma-Fälle in England in den Zeiträumen 2004-2006 und 2007-2009; Quelle: [41].

SWORD = Surveillance of Work-related and Occupational Respiratory Diseases; IIDB = Industrial Injuries Disablement Benefit.

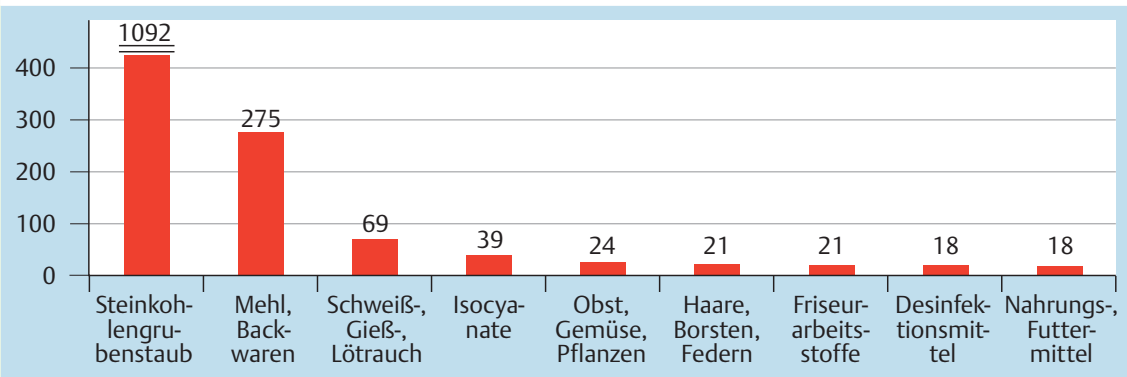

Abb. 2 Vorherrschende ursächliche Stoffe der bestätigten Berufskrankheiten der Atemwege im Zuständigkeitsbereich der gewerblichen Berufsgenossenschaften Deutschlands 2010 (BK 4301, 4302, 1315, 4111), [42].

Tab. 2 Häufigkeit des Berufsasthmas durch einzelne Noxen in Südafrika [20].

\begin{tabular}{|lcc|}
\hline Noxe & Frequenz & $\%$ \\
\hline Latex & 47 & 24,1 \\
\hline Isocyanate & 38 & 19,5 \\
\hline Platin-Salze & 24 & 12,3 \\
\hline Mehl, Getreide & 23 & 11,8 \\
\hline Gesamt & 195 & 100 \\
\hline
\end{tabular}

Mit der Verordnung (EG) Nr.1272/2008 trat am 20.1.2009 das von den Vereinten Nationen festgelegte sogenannte Globally Harmonized System (GHS) of Classification and Labeling of Chemicals [21 - 23]; europäische Bezeichnung: CLP, Classification, Labeling and Packaging of Chemical Products) in Kraft. Dieses ist für Stoffe seit dem 1.12.2010 und für Gemische ab dem 1.6.2015 bindend. Damit gehen neue Kennzeichnungen von Gefahrstoffen einher. Die bisherigen orangefarbenen Gefahrensymbole werden durch auf der Spitze stehende quadratische Gefahren-Piktogramme mit rotem Rand abgelöst [24-26]; H(Hazard Statement)-Sätze lösen die früheren R-Sätze ab. Ein Beispiel ist H334: Es „kann beim Einatmen Allergie, asthmaartige Symptome oder Atembeschwerden verursachen“ (früher R42). Der durch die Hersteller erfolgenden Zuordnung der H-Sätze liegen nicht immer gesicherte wissenschaftliche Erkenntnisse zugrunde.

\section{Weitergehende Kennzeichnung atemwegsrelevanter Gefahrstoffe und andere neue Regularien}

Eine mit der TRBA/TRGS 406 vergleichbare Auflistung atemwegsirritativ oder - toxisch wirkender Arbeitsstoffe gibt es bislang von staatlicher Seite nicht; die deutsche gesetzliche Unfallversicherung (DGUV) fasst in der Handlungsanleitung für arbeitsmedizinische Vorsorge BGI/GUV-I 504-23g [27] entsprechende Expositionen zusammen.
Tab. 3 Obstruktive Atemwegserkrankungen als Berufskrankheiten $2010 \mathrm{im}$ Zuständigkeitsbereich der gewerblichen Berufsgenossenschaften Deutschlands (Anzeigen/bestätigte Fälle), [42].

\begin{tabular}{|lll|}
\hline BK-Nr.4301 & Allergene & $(1930 / 511)$ \\
\hline BK-Nr.4302 & $\begin{array}{l}\text { chem.- irritative oder toxische } \\
\text { Arbeitsstoffe }\end{array}$ & $(1489 / 193)$ \\
\hline BK-Nr.1315 & Isocyanate & $(119 / 39)$ \\
\hline BK-Nr.4111 & Steinkohlengrubenstaub & $(1076 / 1092)$ \\
\hline BK-Nr.4202 & Baumwollstaub & $(12 / 0)$ \\
\hline
\end{tabular}

Weitere für die Atemwege relevante H-Sätze sind: H335 „kann die Atemwege reizen (bisher R37)“, H332 „gesundheitsschädlich beim Einatmen“, H331 „giftig beim Einatmen“, H330 „Lebensgefahr beim Einatmen“. Die zusätzlichen Signalwörter „Gefahr“ und „Achtung“ differenzieren zwischen der schwerwiegenden und der weniger schwerwiegenden Gefahrenkategorie.

Ebenfalls auf der CLP-Verordnung basieren die sog. P-Sätze (Precautionary Statements; früher S-Sätze). Im Kontext mit atemwegsschädigenden Arbeitsstoffen sind die in $\bullet$ Tab. 6 dargestellten P-Sätze von Bedeutung.

Die Einstufung der Stoffe und Gemische erfolgt bis zum 1.6.2015 nach den bisherigen Richtlinien.

Die am 1.12.2010 in Kraft getretene neue Gefahrstoffverordnung [25] berücksichtigt sowohl die CLP- als auch die REACH-Verordnung der EU; neu aufgenommen wurde ferner, dass Tätigkeiten u. a. mit atemwegssensibilisierenden Stoffen und Zubereitungen nur von fachkundigen und besonders unterwiesenen Personen ausgeführt werden dürfen ( $§ 8$ Abs.7).

Ein Link zu einer Zusammenfassung von Arbeitsstoffen mit Effekten auf die Atemwege, basierend auf den Angaben der American Conference of Governmental Industrial Hygienists $\left(\mathrm{ACGIH}^{\circledR}\right)$ und der EU, findet sich in den weiterführenden Informationen. 
Tab.4 Auszug aus der Liste der MAK-Werte 2011 „Liste der Allergene“ Stoffe mit der Bezeichnung Sa und Sah.

\begin{tabular}{|c|}
\hline Alkalipersulfate (Sah) \\
\hline Ammoniumpersulfat [7727-54-0] (Sah) \\
\hline$\alpha$-Amylase (Sa) \\
\hline Beryllium [7440-41-7] und seine anorganischen Verbindungen (Sah) \\
\hline Bromelain [9001-00-7] (Sa) \\
\hline Cellulasen (Sa) \\
\hline Cobalt und Cobaltverbindungen (Sah) \\
\hline 1,2-Diaminoethan [107-15-3] (Sah) \\
\hline Diphenylmethan-4,4'-diisocyanat [101-68-8] (Sah) \\
\hline $\begin{array}{l}\text { Getreidemehlstäube (Sa): } \\
\text { - Roggen, Weizen }\end{array}$ \\
\hline Glutardialdehyd [111-30-8] (Sah) \\
\hline $\begin{array}{l}\text { Hartmetall, Wolframcarbid- und Cobalt-haltig (einatembare Fraktion) } \\
\text { (Sah) }\end{array}$ \\
\hline Hexahydrophthalsäureanhydrid [85-42-7] (Sa) \\
\hline Hexamethylendiisocyanat [822-06-0] (Sah) \\
\hline $\begin{array}{l}\text { Hölzer: } \\
\text { - Terminalia superba Engl. u. Diels, Fraké, Limba (Sa) } \\
\text { - Thuja plicata (D.Don.) Donn., Riesenlebensbaum, Rotzeder, } \\
\text { Western Red Cedar (Sah) } \\
\text { - Triplochiton scleroxylon K.Schum., Abachi, Obeche (Sah) }\end{array}$ \\
\hline Maleinsäureanhydrid [108-31-6] (Sah) \\
\hline Isophorondiisocyanat [4098-71-9] (Sah) \\
\hline Methyltetrahydrophthalsäureanhydrid [11070-44-3] (Sa) \\
\hline 1,5-Naphthylendiisocyanat [3173-72-6] (Sa) \\
\hline Naturgummilatex [9006-04-6] (Sah) \\
\hline Nickel und Nickelverbindungen (Sah) \\
\hline Nickellegierungen (Sah) \\
\hline Papain [9001-73-4] (Sa) \\
\hline Pepsin $[9001-75-6](\mathrm{Sa})$ \\
\hline Phenylisocyanat [103-71-9] (Sah) \\
\hline Phthalsäureanhydrid [85-44-9] (Sa) \\
\hline Phytasen (Sa) \\
\hline Piperazin [110-85-0] (Sah) \\
\hline Platinverbindungen (Chloroplatinate) (Sah) \\
\hline „polymeres MDI“ [9016-87-9] (Sah) \\
\hline Rizinusproteine (Sa) \\
\hline Sojabohneninhaltsstoffe (Sa) \\
\hline Subtilisine $[9014-01-1]$ (Sa) \\
\hline Tierhaare, -epithelien und andere Stoffe tierischer Herkunft (Sah) \\
\hline $\begin{array}{l}\text { Toluylendiisocyanat (Diisocyanattoluol) (Sa): } \\
\text { - 2,4-Toluylendiisocyanat [584-84-9] } \\
\text { - 2,6-Toluylendiisocyanat [91-08-7] }\end{array}$ \\
\hline Trimellitsäureanhydrid [552-30-7] (Sa) \\
\hline Xylanasen [37278-89-0] (Sa) \\
\hline Zirkonium [7440-67-7] und seine unlöslichen Verbindungen (Sah) \\
\hline Zirkoniumverbindungen (löslich) (Sah) \\
\hline
\end{tabular}

\section{Diagnostik}

$\nabla$

Dem diagnostischen Prozedere liegt ein Stufenschema zugrunde ( Abb.3). Dabei kommt der detaillierten Arbeitsanamnese, die stets die klinische Anamnese ergänzt, eine besondere Bedeutung zu. Arbeitsbedingte allergische und irritative Atemwegserkrankungen weisen typischerweise einen Bezug der Beschwerden zur Tätigkeit auf. Am Feierabend und v.a. während arbeitsfreier Phasen (Wochenende, Urlaub, Arbeitsunfähigkeit) bilden sich die Beschwerden im Frühstadium zurück. Die Latenzzeit zwischen Tätigkeits(Expositions)-Beginn und dem Auftreten erster Symptome ist sehr variabel. Im Falle allergischer Pathomechanismen beträgt sie mindestens mehrere Wochen (Sensibilisierungs-
Tab. 5 Anlage zur TRBA/TRGS 406 „Sensibilisierende Stoffe für die Atemwege" " [43]). Berücksichtigt sind Stoffe, welche nach Anhang I der Richtlinie $67 / 548 /$ EWG mit „R42“ oder „R42/43“ oder nach MAK- und BAT-Werteliste mit „Sa“ oder "Sah" markiert sind.

Chemische Stoffe
- Azodicarbonamid
- Beryllium
- Chloramin T
- Kobalt und seine Verbindungen
- Dicarbonsäureanhydride
- Enzyme
- Ethylendiamin
- Glutardialdehyd
- Isocyanate
- Nickel und seine Verbindungen
- Persulfate
- Piperazin
- Platinverbindungen
Pflanzliche Stoffe
- Getreide und Futtermittel (verschiedene)
- Hölzer (verschiedene), Abachi, Limba
- Mehl (Weizenmehl, Roggenmehl u. a.)
- Naturgummilatex
- Zier- und Nutzpflanzen (bzw. Teile davon)
- Tee
- Kaffeebohnen
- Unkräuter
Tierische Stoffe
- Haare, Borsten, Federn, Horn, Kot, Urin
- Fische und Schalentiere
- Milben (Vorrats-, Hausstaub-, Spinnenmilben)
- Zuckmückenlarven
Biologische Arbeitsstoffe
- Bakterien wie thermophile Actinomyceten
- Schimmelpilze wie Aspergillus spp., Penicillium spp.,
Cladosporium spp., Alternaria spp.
Sonstige Stoffe
- Antibiotika

Tab.6 CLP-Verordnung: Kennzeichnung durch P-Sätze (früher S) [44].

\begin{tabular}{|c|c|}
\hline P260 & Staub, Rauch, Gas, Nebel, Dampf, Aerosol nicht einatmen \\
\hline P261 & $\begin{array}{l}\text { Einatmung von Staub, Rauch, Gas, Nebel, Dampf, Aerosol } \\
\text { vermeiden }\end{array}$ \\
\hline P284 & Atemschutz tragen \\
\hline P285 & Bei unzureichender Belüftung Atemschutz tragen \\
\hline P340 & $\begin{array}{l}\text { Die betroffene Person an die frische Luft bringen und in einer } \\
\text { Position ruhigstellen, die das Atmen erleichtert }\end{array}$ \\
\hline P341 & $\begin{array}{l}\text { Bei Atembeschwerden an die frische Luft tragen und in einer } \\
\text { Position ruhigstellen, die das Atmen erleichtert }\end{array}$ \\
\hline P342 & $\begin{array}{l}\text { Bei Symptomen der Atemwege: Nur in Kombination mit weiterer } \\
\text { Angabe sinnvoll, z. B. P311 = Giftinformationszentrum oder Arzt } \\
\text { anrufen }\end{array}$ \\
\hline
\end{tabular}

phase), sie kann sich auch über viele Jahre erstrecken. Eine hohe Exposition gegenüber Irritanzien kann akut das Krankheitsbild initiieren; in der anglosächsischen Literatur hatte sich hierfür der Begriff „RADS“ (Reactive Airways Dysfunction Syndrome) eingebürgert, der in neuerer Zeit durch „Irritant-induced asthma“ ersetzt wird.

Die durch niedrigere langjährige Belastungen durch Irritanzien, auch Schweißrauche und anorganische Stäube, verursachte COPD entwickelt sich schleichend und manifestiert sich in Form einer progredienten Belastungsdyspnoe. 


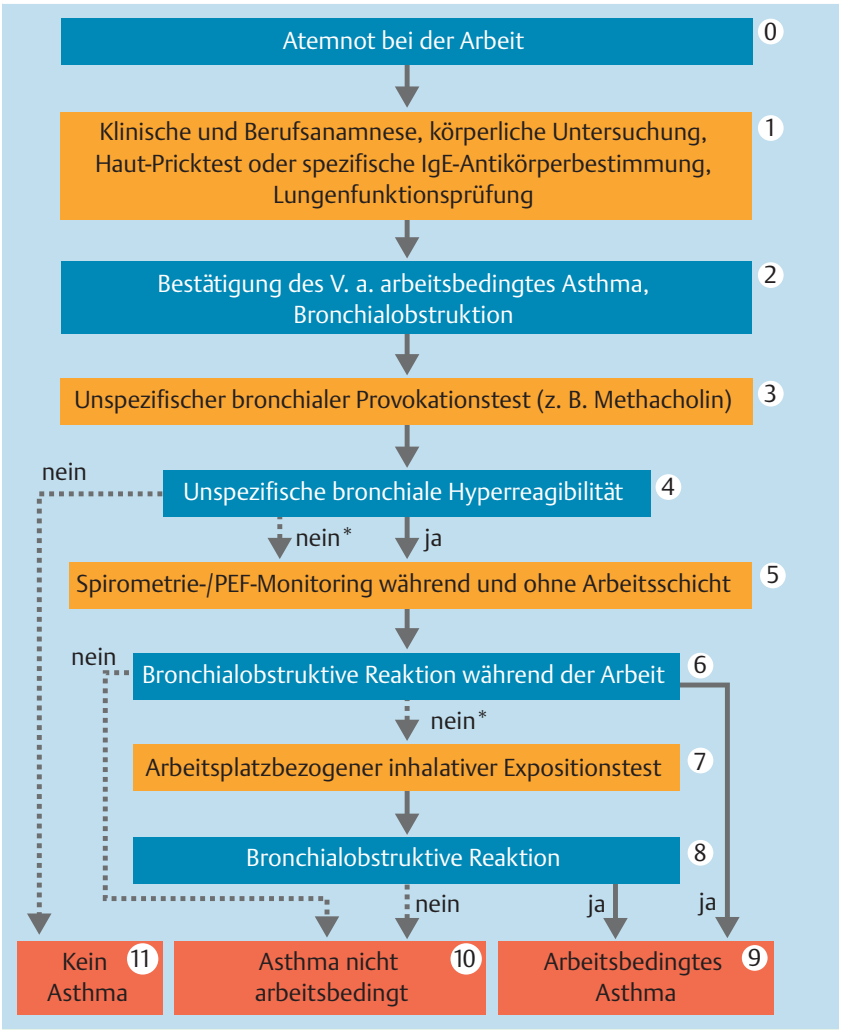

Abb.3 Diagnostisches Stufenschema bei Verdacht auf arbeitsbedingtes Asthma bronchiale.

* Es gibt Ausnahmen, z. B. beim Isocyanat-bedingten Asthma, sodass hier die dargestellte weitergehende Diagnostik indiziert sein kann.

Die körperliche Untersuchung ergibt beim Asthmaerkrankten initial im Intervall, das heißt außerhalb der akuten Belastung gegenüber der ursächlichen Noxe, keine Auffälligkeiten. Entsprechendes gilt für die Lungenfunktionsprüfung. Bereits im Frühstadium kommt dem Methacholintest, der Erfassung der unspezifischen bronchialen Hyperreaktivität, eine hohe Sensitivität (vergleiche Fallbeispiel in $\bigcirc$ Abb.4) bei allerdings niedriger Spezifität zu. Im Karenz- und Reexpositionsversuch, den der Betriebsarzt am besten zusammen mit dem betreuenden Hausarzt oder Pneumologen durchführt, lässt sich die ursächliche Bedeutung eines bestimmten Tätigkeitsbereichs oder eines bestimmten Arbeitsstoffes für das vorliegende Asthmaleiden oft eindrucksvoll belegen. Zur Objektivierung erfolgen serielle Lungenfunktionsmessungen vor der Arbeitsschicht, zweistündlich während derselben und danach; gleichartige Messungen werden an arbeitsfreien Tagen durchgeführt. Eine reproduzierbare Verschlechterung der FEV1 oder des PEF ( $\geq 20 \%$ ) während der Tätigkeit hat eine hohe diagnostische Wertigkeit $[2,28]$.

Die Allergiediagnostik dient der Objektivierung bzw. dem Ausschluss einer Typ I-Sensibilisierung. Hierzu ist es erforderlich, von den Arbeitsstoffen standardisierte Extrakte für den HautPrick-Test oder für die Bestimmung spezifischer IgE-Antikörper einzusetzen.

Spezifische inhalative Provokationsteste mit Allergenextrakten und arbeitsplatzbezogene inhalative Expositionsteste sind Problemfällen in spezialisierten Zentren vorbehalten. Sie gelten als Goldstandard der Diagnostik des arbeitsbedingten Asthma bronchiale [29-32] (s. Beispiel in $\odot$ Abb.4).

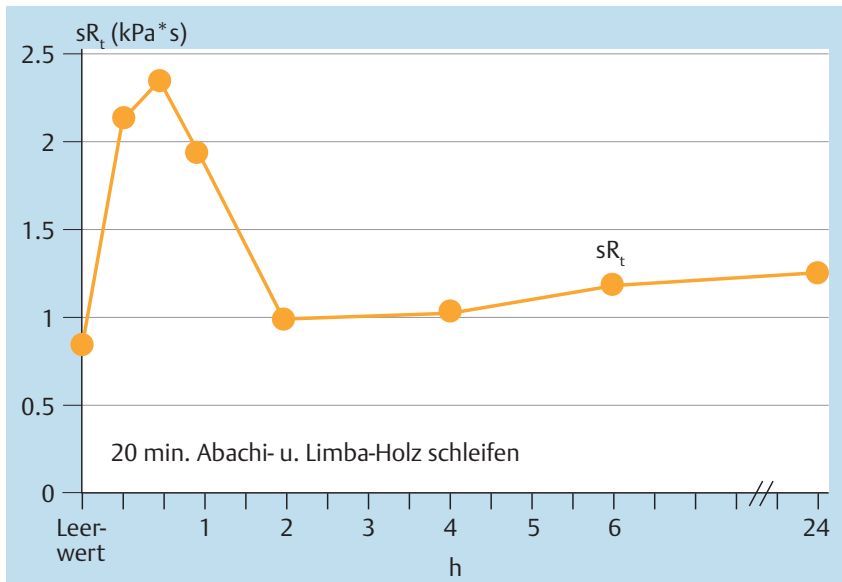

Abb.4 Arbeitsplatzbezogener Expositionstest (20-minütiges Schleifen von Abachi- und Limbaholz). $\mathrm{sR}_{\mathrm{t}}=$ spezifischer Atemwegswiderstand, ganzkörperplethysmografisch bestimmt.

Anamnese und Lungenfunktion des 32-jährigen Schreiners:

Berufsanamnese: Seit 10 Jahren Schreinertätigkeit. Dabei Umgang mit verschiedenen einheimischen Hölzern sowie mit Limba- und Abachi-Holz. Klinische Anamnese: Seit 3 Jahren im Rahmen des berufsbedingten Kontakts mit Staub der exotischen Hölzer Fließschnupfen, Niesattacken, Hustenreiz, seit einigen Monaten zunehmend auch thorakales Beklemmungsgefühl.

Lungenfunktionsbefunde: Unter Ruhebedingungen unauffällig. Im Methacholinprovokationstest vor einem Jahr erstmals Zeichen einer bronchialen Hyperreaktivität, die in der jetzigen Kontrolle stark zugenommen hat (Methacholindosis, die zu einem 20\%igen FEV1-Abfall führte, initial 0,2 mg, zuletzt $0,01 \mathrm{mg}$ ).

\section{Differenzialdiagnosen}

$\nabla$

Wichtigste Differenzialdiagnosen umfassen andere arbeitsbedingte Erkrankungen wie exogen allergische Alveolitis, Pneumokoniosen, toxisches Lungenödem, Metallrauchfieber, Organic dust toxic syndrome (ODTS), aber auch das Intrinsic Asthma, Asthma durch ubiquitäre Allergene, allergische bronchopulmonale Aspergillose, pulmonale Vaskulitis, zystische Fibrose, Lungenemphysem, Trachealstenose, okklusives Schlafapnoesyndrom, Vocal cord dysfunction syndrome (VCD). Die eindeutige Diagnosestellung ist wegen der i.d.R. abweichenden Therapie und Prävention erforderlich. Zur Abgrenzung, insbesondere von nicht arbeitsbedingten chronischen Erkrankungen der Nasennebenhöhlen, der Haut oder einer gastroösophagealen Refluxkrankheit, ist das betreffende medizinische Fachgebiet einzubeziehen. Ein HRCT des Thorax kann bei Verdacht auf einen interstitiellen Lungenprozess oder ein Lungenemphysem indiziert sein. Neuere Untersuchungen wie jene des induzierten Sputums, des Atemkondensats, der exhalierten NO-Fraktion (FeNO) und der Nasal-Lavage sind hilfreich, jedoch bisher nur teilweise standardisiert.

\section{Prävention \\ $\nabla$}

Bezüglich der stets anzustrebenden Primärprävention, also der Vermeidung der gesundheitsgefährdenden Exposition, z. B. durch Substitution der Noxe, Einsatz geschlossener Produktionstechnologien etc., wird auf die Leitlinie „Prävention arbeitsbedingte obstruktive Atemwegserkrankungen“ verwiesen [33].

Falls durch technische und organisatorische Maßnahmen eine Gefährdung der Atemwege durch sensibilisierende, chemisch-ir- 
ritativ oder toxisch wirkende Stoffe oder hohe Konzentrationen anorganischer Stäube nicht vermieden werden kann, sollten die Beschäftigten regelmäßigen arbeitsmedizinischen Vorsorgeuntersuchungen unterzogen werden. In der Verordnung zur arbeitsmedizinischen Vorsorge [34] werden darüber hinaus die Anlässe für Pflicht- und Angebotsuntersuchungen festgelegt (๑ Tab. 7).

Die Handlungsanleitungen BGI/GUV-I 504-X [35] für die arbeitsmedizinischen Vorsorgeuntersuchungen G 1.4 Staubbelastung, G 23 Obstruktive Atemwegserkrankungen [27,36], G 27 Isocyanate sowie G 39 Schweißrauche geben zusätzliche Hinweise zu stoffspezifischen Kriterien für die Risikobewertung und die Auswahl der zu Untersuchenden.

In der Arbeitsmedizinischen Vorsorgeverordnung [34] wird Mehlstaub in der Liste der Stoffe, bei denen Pflichtuntersuchungen vorgesehen sind, aufgeführt. Allerdings wurde bislang in der TRGS 900 kein Arbeitsplatzgrenzwert festgelegt [37]. Aus diesem rein formalen Grund sind derzeit keine Pflichtuntersuchungen zu veranlassen, auch wenn sie bei hoher Mehlstaubkonzentration inhaltlich gut begründbar sind; s.a. Handlungsanleitung für arbeitsmedizinische Vorsorge BGI/GUV-I 504-23 h [36].

Für die Durchführung von Vorsorgeuntersuchungen und die diesbezügliche ärztliche Beurteilung sind als anerkannte Regeln der Arbeitsmedizin die DGUV-Grundsätze für arbeitsmedizinische Vorsorgeuntersuchungen heranzuziehen [38]. Eine Liste der Grundsätze findet man unter http://www.ifam.med.uni-rostock. de/bggr.htm.

Wie mehrere Studien belegen, verschlechtert die fortgesetzte ursächliche Exposition des Erkrankten die Prognose [19,39]. Daher ist die strikte Karenz - häufig bedeutet dies einen Arbeitsplatzwechsel - die erfolgversprechendste und wichtigste sekundärpräventive Maßnahme.

Dagegen sind eine reduzierte Belastung und das Tragen von Atemschutz keine Garanten für einen günstigen Verlauf [40].

Da Rauchen für sich alleine schon zu einer Verschlechterung der Lungenfunktion führt, sollten neben der individuellen Beratung auch Antiraucherkampagnen in den Betrieben initiiert werden.

Darüber hinaus sind Patientenschulungen mit besonderer Betonung von Strategien zur Vermeidung arbeitsbedingter Trigger, der Erkennung von Exazerbationen und symptomorientierter Optimierung der medikamentösen Therapie erforderlich. Letztere unterscheidet sich nicht von Atemwegserkrankungen, die nicht auf arbeitsbedingte Ursachen zurückgehen. Eine Hyposensibilisierung gegen Berufsallergene wird nicht empfohlen, da der Erfolg einer solchen Behandlung nicht belegt ist.

Abschließend sei auf §202 der Berufskrankheitenverordnung verwiesen, der jeden Arzt verpflichtet, den begründeten Verdacht auf eine Berufskrankheit dem zuständigen Unfallversicherungsträger (Berufsgenossenschaft) oder der staatlichen Stelle für den Arbeitsschutz (Gewerbearzt) per Formblatt (s. unter „Weiterführende Informationen“) zu melden. Entsprechendes gilt für den Arbeitgeber; auch Betroffene können selbst den Verdacht auf eine Berufskrankheit melden.

Ferner ist $\S 3$ der Berufskrankheitenverordnung zu berücksichtigen; demnach muss der konkreten Gefahr der Entstehung einer Berufskrankheit mit allen geeigneten Mitteln entgegengewirkt werden. Dies ist auch für den Fall einer noch nicht manifesten Berufskrankheit relevant, wenn z. B. ein präklinischer Zustand infolge arbeitsbedingter Exposition oder eine höhergradige Sensibilisierung bei noch fehlender eindeutiger Funktionseinschränkung vorliegt. Maßnahmen im Sinne des $§ 3$ der BKV sind auch dann indiziert, wenn bei einem vorbestehenden oder anlagebedingten
Tab. 7 Anhang Arbeitsmedizinische Pflicht- und Angebotsuntersuchungen sowie weitere Maßnahmen der arbeitsmedizinischen Vorsorge (Auszug) [45].

\section{Teil 1 Tätigkeiten mit Gefahrstoffen}

\section{(1) Pflichtuntersuchungen bei:}

1. Tätigkeiten mit den Gefahrstoffen:

Alveolengängiger Staub (A-Staub)

Asbest

Beryllium

Cadmium und Cadmiumverbindungen

Chrom-VI-Verbindungen

Dimethylformamid

Einatembarer Staub (E-Staub)

Fluor und anorganische Fluorverbindungen

Hartholzstaub

Mehlstaub

Nickel und Nickelverbindungen

Platinverbindungen

Silikogener Staub

Trichlorethen

2. Sonstige Tätigkeiten mit Gefahrstoffen:

Schweißen und Trennen von Metallen bei Überschreitung einer Luftkonzentration von 3 Milligramm pro Kubikmeter Schweißrauch Tätigkeiten mit Exposition gegenüber Getreide- und Futtermittelstäuben bei Überschreitung einer Luftkonzentration von $4 \mathrm{mg} / \mathrm{m}^{3}$ einatembarem Staub

Tätigkeiten mit Exposition gegenüber Isocyanaten, bei denen ein regelmäßiger Hautkontakt nicht vermieden werden kann oder eine Luftkonzentration von $0,05 \mathrm{mg} / \mathrm{m}^{3}$ überschritten wird

Tätigkeiten mit einer Exposition mit Gesundheitsgefährdung durch Labortierstaub in Tierhaltungsräumen und -anlagen

Tätigkeiten mit Benutzung von Naturgummilatexhandschuhen mit mehr als $30 \mu \mathrm{g}$ Protein je Gramm im Handschuhmaterial Tätigkeiten mit dermaler Gefährdung oder inhalativer Exposition mit Gesundheitsgefährdung, verursacht durch unausgehärtete Epoxidharze

(2) Angebotsuntersuchungen bei:

1. Tätigkeiten mit den in Absatz 1 Nr. 1 genannten Gefahrstoffen, wenn eine Exposition besteht;

2. Sonstige Tätigkeiten mit Gefahrstoffen:

a) Schädlingsbekämpfung nach Anhang I Nummer 3 der Gefahrstoffverordnung,

b) Begasungen nach Anhang I Nummer 4 der Gefahrstoffverordnung,

c) Tätigkeiten mit folgenden Stoffen oder deren Gemischen: n-Hexan, n-Heptan, 2-Butanon, 2-Hexanon, Methanol, Ethanol, 2-Methoxyethanol, Benzol, Toluol, Xylol, Styrol, Dichlormethan, 1,1,1-Trichlorethan, Trichlorethen, Tetrachlorethen,

d) Tätigkeiten mit krebserzeugenden oder erbgutverändernden Stoffen oder Zubereitungen der Kategorie 1 oder 2 im Sinne der Gefahrstoffverordnung,

e) Feuchtarbeit von regelmäßig mehr als zwei Stunden je Tag,

f) Schweißen und Trennen von Metallen bei Einhaltung einer Luftkonzentration von 3 Milligramm pro Kubikmeter Schweißrauch,

g) Tätigkeiten mit Exposition gegenüber Getreide- und Futtermittelstäuben bei Überschreitung einer Luftkonzentration von 1 Milligramm je Kubikmeter einatembarem Staub.

3. Untersuchungen nach den Nummern 1 und 2 müssen nicht angeboten werden, wenn nach der Gefährdungsbeurteilung die Voraussetzungen des $\S 6$ Absatz 11 der Gefahrstoffverordnung vorliegen und die nach $\S 8$ der Gefahrstoffverordnung ergriffenen Maßnahmen zum Schutz der Beschäftigten ausreichen.

Leiden durch die arbeitsbedingte Exposition eine wesentliche Verschlimmerung droht.

\section{Interessenkonflikt}

$\nabla$

Der Autor gibt an, dass kein Interessenkonflikt besteht. 


\section{Literatur}

1 Balmes J, Becklake M, Blanc P et al. American Thoracic Society Statement: Occupational contribution to the burden of airway disease. Am J Respir Crit Care Med 2003; 167: 787-797

2 Anees W, Gannon PF, Huggins Vet al. Effect of peak expiratory flow data quantity on diagnostic sensitivity and specificity in occupational asthma. Eur Respir J 2004; 23: 730 - 734

3 Kogevinas M, Anto JM, Sunyer J et al. Occupational asthma in Europe and other industrialised areas: a population-based study. European Community Respiratory Health Survey Study Group. Lancet 1999; 353: $1750-1754$

4 Oxman $A D$, Muir DCF, Shannon HS et al. Occupational dust exposure and chronic obstructive pulmonary disease. Am Rev Respir Dis 1993; 148: $38-48$

5 Hnizdo E, Vallyathan $V$. Chronic obstructive pulmonary disease due to occupational exposure to silica dust: a review of epidemiological and pathological evidence. Occup Environ Med 2003; 60: 237-243

6 Cohen RA, Patel A, Green FH. Lung disease caused by exposure to coal mine and silica dust. Semin Respir Crit Care Med 2008; 29: 651 -661

7 Marine WM, Gurr D, Jacobsen M. Clinically important respiratory effects of dust exposure and smoking in British coal miners. Am Rev Respir Dis 1988; 137: 106-112

8 Baur X, Vellguth $H$. Bronchial asthma and COPD due to irritants in the workplace - an evidence-based approach. Int Arch Occup Environ Health [submitted].

9 Baur X. Liste der atemwegsirritativen Arbeitsstoffe. 2011: http://www. uke.de/institute/arbeitsmedizin/downloads/universitaetsprofessur-arbeitsmedizin/Table_2_Irritants.pdf

10 Baur X. Liste der atemwegssensitiven Arbeitsstoffe. 2010: http://www. uke.de/institute/arbeitsmedizin/downloads/universitaetsprofessur-arbeitsmedizin/Table_1_allergenic_agents.pdf

11 Mastruzzo C, Crimi N, Vancheri C. Role of oxidative stress in pulmonary fibrosis. Monaldi Arch Chest Dis 2002; 57: 173-176

12 Schins RP, Borm PJ. Mechanisms and mediators in coal dust induced toxicity: a review. Ann Occup Hyg 1999; 43: 7-33

13 Holgate ST. Epithelium dysfunction in asthma. The Journal of allergy and clinical immunology 2007; 120: 1233 -1244; quiz 1245-1246.

14 Palmer CN, Ismail T, Lee SP et al. Filaggrin null mutations are associated with increased asthma severity in children and young adults. The Journal of allergy and clinical immunology 2007; 120: 64-68

15 Mauad T, Bel EH, Sterk PJ. Asthma therapy and airway remodeling. The Journal of allergy and clinical immunology 2007; 120: 997 - 1009; quiz 1010-1011.

16 Baur X, Clasen M, Fisseler-Eckhoff A et al. Diagnostik und Begutachtung asbestbedingter Berufskrankheiten. Interdisziplinäre S2-Leitlinie der Deutschen Gesellschaft für Pneumologie und Beatmungsmedizin und der Deutschen Gesellschaft für Arbeitsmedizin und Umweltmedizin. Pneumologie 2011; 65: 159-188

17 Sigsgaard T, Nowak D, Annesi-Maesano I et al. ERS position paper: work-related respiratory diseases in the EU. Eur Respir J 2010; 35: $234-238$

18 Bergdahl IA, Toren $K$, Eriksson $K$ et al. Increased mortality in COPD among construction workers exposed to inorganic dust. Eur Respir J 2004; 23: $402-406$

19 Latza U, BaurX. Occupational obstructive airway diseases in Germany: Frequency and causes in an international comparison. Am J Ind Med 2005; 48: $144-152$

20 Hnizdo E, Esterhuizen TM, Rees D et al. Occupational asthma as identified by the Surveillance of Work-related and Occupational Respiratory Diseases programme in South Africa. Clin Exp Allergy 2001; 31: 32 - 39

21 GHS. Das Global Harmonisierte System (GHS) in der EU. Die neue Einstufung und Kennzeichnung nach CLP-Verordnung (EG) Nr. 1272/ 2008. Gefahren- und Sicherheitshinweise. 2011; http://www.baua. de/de/Publikationen/Poster/GHS-02.pdf?__blob=publicationFile $\& v=10$

22 Zhang L, Curran I, Muradia G et al. Two-dimensional immunoblot analysis of allergens of Cladosporium herbarum. Clin Exp Allergy 1994; 24: $263-269$

23 Guyatt G, Cook D, Haynes B. Evidence based medicine has come a long way. Br Med J 2004; 329: 990-991

24 United Nations Economic Commission for Europe (UNECE). GHS pictograms. http://live.unece.org/trans/danger/publi/ghs/pictograms.html

25 Bundesregierung. Verordnung zum Schutz vor Gefahrstoffen (Gefahrstoffverordnung - GefStoffV) vom 26.11.2010. BGBl I (59). 2010:
1644-1676; http://www.baua.de/de/Themen-von-A-Z/Gefahrstoffe/ Rechtstexte/Gefahrstoffverordnung_content.html

26 Bundesanstalt für Arbeitsschutz und Arbeitsmedizin (BAuA). REACHCLP Helpdesk der Bundesbehörden. 2007: http://www.reach-clp-helpdesk.de/reach/de/Startseite.html

27 Deutsche Gesetzliche Unfallversicherung (DGUV). Handlungsanleitung für die arbeitsmedizinische Vorsorge nach dem Berufsgenossenschaftlichen Grundsatz G 23 „Obstruktive Atemwegserkrankungen, hier: Atemwegsreizende Stoffe (chemisch-irritative und chemisch-toxische)“ BGI/GUV-I 504-23g. 2009; http://publikationen.dguv.de/ dguv/pdf/10002/i-504-23g.pdf

28 Moore VC, Jaakkola M, Burge P. A systematic review of serial peak expiratory flow measurements in the diagnosis of occupational asthma. Ann Respir Med 2010; 1: 31 - 44

29 Baur X, Haamann F, Heutelbeck A et al. Arbeitsmedizinische Leitlinien der Deutschen Gesellschaft für Arbeitsmedizin und Umweltmedizin e.V. Arbeitsplatzbezogener Inhalationstest (AIT). Arbeitsmed Sozialmed Umweltmed 2005; 40: 260-267

30 Baur X. [Working place related obstructive airway diseases]. Pneumologie 2006; 60: 305-314; quiz 315-319.

31 Vandenplas 0 , Delwiche JP, Jamart J et al. Increase in non-specific bronchial hyperresponsiveness as an early marker of bronchial response to occupational agents during specific inhalation challenges. Thorax 1996; 51: $472-478$

32 Baur X. [Bronchial Challenge Tests]. Pneumologie 2011; 65: 340 - 346

33 Baur X, Heutelbeck A, Kampen V et al. Prävention arbeitsbedingter obstruktiver Atemwegserkrankungen. Leitlinie der Deutschen Gesellschaft für Arbeitsmedizin und Umweltmedizin (DGAUM). Pneumologie 2010; 65: 263-282

34 ArbMed VV. Verordnung zur arbeitsmedizinischen Vorsorge vom 18. Dezember 2008 (BGBl. I S. 2768), die zuletzt durch Artikel 5 Absatz 8 der Verordnung vom 26. November 2010 (BGBl. I S. 1643) geändert worden ist. http://bundesrecht.juris.de/bundesrecht/arbmedvv/gesamt.pdf; 2008 .

35 Deutsche Gesetzliche Unfallversicherung (DGUV). Handlungsanleitungen für die arbeitsmedizinische Vorsorge. BGI/GUV-I 504. http://publikationen.dguv.de/dguv/udt_dguv_main.aspx?QPX=TUlEPTEwMDEmSUQ9MA\%3d\%3d - Informationen. 2009.

36 Deutsche Gesetzliche Unfallversicherung (DGUV). Handlungsanleitung für die arbeitsmedizinische Vorsorge nach dem Berufsgenossenschaftlichen Grundsatz G 23 „Obstruktive Atemwegserkrankungen, hier: Atemwegssensibilisierende Stoffe und Stoffgruppen“. BGI/GUV-I 504-23h. 2009: http://www.euk-info.de/fileadmin/PDF_Archiv/Regelwerk_Archiv/Info_arb_Vorsorge/GUV-I_504-23h.pdf

37 Ausschuss für Gefahrstoffe (AGS). Arbeitsplatzgrenzwerte (TRGS 900) (aktualisiert 2010). GMBl 2006; 43: 912-913; http://www.baua.de/ cln_137/de/Themen-von-A-Z/Gefahrstoffe/TRGS/TRGS-900.html

38 Deutsche Gesetzliche Unfallversicherung (DGUV). DGUV Grundsätze für arbeitsmedizinische Vorsorgeuntersuchungen. 5. Auflage. Stuttgart: Gentner Verlag; 2010

39 Maestrelli P, Schlünssen V, Masson P et al. Contribution of Horst Factors and Workplace Exposure to the Outcome of Occupational Asthma. Eur Respir Rev 2011: [in press].

40 Baur X, Aasen T, Burge $S$ et al. Chapter 5 of ERS guidelines „Management of work-related asthma“: The „Management of Work-related Asthma“ guidelines in a broader perspective. Eur Respir Rev [in press].

41 Health and Safety Executive (HSE). ed. Occupational Asthma. Causal agents for occupational asthma. http://www.hse.gov.uk/statistics/ causdis/asthma/agents.htm

42 Deutsche Gesetzliche Unfallversicherung (DGUV). BK-DOK 2010. Persönliche Mitteilung. 2011

43 Ausschuss für Biologische Arbeitsstoffe (ABAS), Ausschuss für Gefahrstoffe (AGS). Sensibilisierende Stoffe für die Atemwege (TRBA/TRGS 406). 2008; http://www.baua.de/de/Themen-von-A-Z/Gefahrstoffe/ TRGS/pdf/TRGS-TRBA-406.pdf?__blob=publicationFile\&v=3)

44 United Nations. Codification of Hazard Statements, Codification and use of Precautionary Statements and Examples of Precautionary Pictograms. In: Globally Harmonized System of Classification and Labelling of Chemicals (GHS). Third revised edition. New York, Geneva: United Nations; 2009: 297 -401; http://live.unece.org/fileadmin/DAM/trans/ danger/publi/ghs/ghs_rev203/English/207e_annex293.pdf

45 Bundesregierung. Anhang. Arbeitsmedizinische Pflicht- und Angebotsuntersuchungen sowie weitere Maßnahmen der arbeitsmedizinischen Vorsorge (Auszug). BGBl I 2008: 2771-2775 


\section{Weitere hilfreiche Links}

46 Leitlinie „Lungenfunktionsprüfungen in der Arbeitsmedizin“. http://www.awmf.org/uploads/tx_szleitlinien/002 -013_S1_Lungenfunktionspruefungen_in_der_Arbeitsmedizin_08-2005_08-2010.pdf 47 Leitlinie „Arbeitsplatzbezogener Inhalationstest (AIT)“. http://www.awmf.org/uploads/tx_szleitlinien/002-026_S1_Arbeitsplatzbezogener_Inhalationstest_AIT_11_2009_11-2011.pdf
48 Ärztliche Anzeige bei Verdacht auf eine Berufskrankheit. http://arbmed.med.uni-rostock.de/bkvo/BK_Arzt.pdf

49 Anzeige des Unternehmers bei Anhaltspunkten für eine Berufskrankheit. http://arbmed.med.uni-rostock.de/bkvo/BK_Betrieb.pdf

50 Tabelle der von ACGIH und EU als atemwegssensibilisierend oder-irritativ eingestuften Arbeitsstoffe. http://www.uke.de/institute/arbeitsmedizin/downloads/universitaetsprofessur-arbeitsmedizin/ R42_und_R37A-EU09.pdf 